\title{
Review of: "Prescribing of direct oral anticoagulants and warfarin to older people with atrial fibrillation in UK general practice: a cohort study"
}

Pietro Scicchitano

Potential competing interests: The author(s) declared that no potential competing interests exist.

Title: “Prescribing of direct oral anticoagulants and warfarin to older people with atrial fibrillation in UK general practice: a cohort study"

Dear Editor,

I read this paper and I think that:

- The retrospective nature of this study is a limitation and should be discussed in a dedicated limitation section.

- The authors tried to investigate the persistence on DOAC/Warfarin. Indeed, the authors considered a study period between 1st January 2003 to 27th December 2017. Therefore, lots of patients could not be on DOACs between at least 2003 and 2009. This should be considered and analysed when dealing with persistence.

- No mention is for cancer incidence. May the authors provide data about the incidence of cancer and the relationship among cancer, AF, anticoagulation, and DOACs?

- One of the most intriguing considerations about "real world" data on DOACs is the role of bridging therapy on the overall results. The LMWHs which are prescribed before attaining the correct INR value impact on prescription, adherence, and - to some extent - persistence on Warfarin. Please discuss such a point. 